\title{
REVIEWS
}

\section{A Systematic Review of Communication Quality Improvement Interventions for Patients with Advanced and Serious IIIness}

\author{
Oluwakemi A. Fawole, MBChB, MPH' , Sydney M. Dy, MD, MSc. ${ }^{2}$, Renee F. Wilson, M.S. ${ }^{3}$, \\ Brandyn D. Lau, MPH4, Kathryn A. Martinez, PhD ${ }^{5}$, Colleen C. Apostol, MSN, RN', \\ Daniela Vollenweider, $\mathrm{MD}^{7}$, Eric B. Bass, MD, $\mathrm{MPH}^{8}$, and Rebecca A. Aslakson, MD, MSc. ${ }^{9}$ \\ 'Johns Hopkins University Evidence-Based Practice Center, Baltimore, MD, USA; 2 Department of Health Policy \& Management, Oncology, \\ and Medicine,Johns Hopkins University, Baltimore, MD, USA; ${ }^{3}$ Johns Hopkins University Evidence-Based Practice Center, Baltimore, MD, USA; \\ ${ }^{4}$ Evidence-Based Practice Center, Johns Hopkins University, Baltimore, MD, USA; ${ }^{5}$ Department of Health Policy and Management, Johns \\ Hopkins Bloomberg School of Public Health, Baltimore, MD, USA; 'Duffey Pain and Palliative Care Program, Johns Hopkins Hospital, Baltimore, \\ MD, USA; ${ }^{7}$ Division of General Internal Medicine, Johns Hopkins University, Baltimore, MD, USA; ${ }^{8}$ Johns Hopkins University Evidence-Based \\ Practice Center, Baltimore, MD, USA; ${ }^{9}$ Department of Anesthesiology and Critical Care Medicine, Johns Hopkins School of Medicine, \\ Baltimore, MD, USA.
}

BACKGROUND: Effective communication is an interaction between two or more people that produces a desired effect and is a key element of quality of care for patients with advanced and serious illness and their family members. Suboptimal provider-patient/family communication is common, with negative effects on patient/family-centered outcomes.

OBJECTIVES: To systematically review the evidence for effectiveness of communication-related quality improvement interventions for patients with advanced and serious illness and to explore the effectiveness of consultative and integrative interventions.

DATA SOURCES: MEDLINE, CINAHL, PsycINFO, Cochrane, and DARE from 2000 through December 2011 and reference list of eligible articles and reviews.

STUDY ELIGIBILITY CRITERIA, PARTICIPANTS AND INTERVENTIONS: Prospective, controlled quality improvement studies in populations with life-limiting or severe life-threatening illness with a primary intervention focus of improving communication with patients and/or families.

STUDY APPRAISAL AND SYNTHESIS METHODS: Two investigators independently screened and abstracted data on patient/family-centered outcomes.

RESULTS: We included 20 studies; 13 (65\%) were in intensive care. We found four intervention types: (1) family meetings with the usual team (11 studies, $77 \%$ found improvement in healthcare utilization), (2) palliative care teams (5 studies, $50 \%$ found improvement in healthcare utilization), (3) ethics consultation (2 studies, $100 \%$ found improvement in healthcare utilization), and (4) physician-patient communication (2 studies, no significant improvement in healthcare utilization). Among studies addressing the outcomes of patient/family satisfaction, $22 \%$ found improvement; among studies addressing healthcare utilization (e.g., length of stay), $73 \%$ found improvement. Results

Received April 20, 2012

Revised July 19, 2012

Accepted July 23, 2012

Published online October 26, 2012 suggest that consultative interventions, as opposed to integrative ones, may be more effective, but more research is needed.

LIMITATIONS: Study heterogeneity did not allow quantitative synthesis.

CONCLUSIONS AND IMPLICATIONS OF KEY FINDINGS: Communication in the care of patients with advanced and serious illness can be improved using quality improvement interventions, particularly for healthcare utilization as an outcome. Interventions may be more effective using a consultative approach.

KEY WORDS: communication; seriously ill patients; patients with advanced diseases; quality improvement interventions.

$\mathrm{J}$ Gen Intern Med 28(4):570-7

DOI: $10.1007 /$ s1 1606-012-2204-4

(C) Society of General Internal Medicine 2012

\section{INTRODUCTION}

Effective communication between providers and patients and their families is essential in palliative care. Communication involves sharing information, thoughts and feelings between two or more people, and it is effective when the interaction conveys the intended message. Thus, the quality of communication in the care of patients with advanced and serious illness is a key determinant of patient and family outcomes. Research in a variety of settings has found that communication is often suboptimal. For example, in a study of audiotaped initial oncology consultations for patients with advanced cancer, fewer than half of oncologists offered alternatives to chemotherapy as an option, only $58 \%$ of patients were informed of their life expectancy, and only $60 \%$ were aware of risks versus benefits of further chemotherapy treatment. ${ }^{1}$ This informational gap can affect patient/family-centered outcomes, which are outcomes important to the patient/family, such as patient satisfaction, family satisfaction, length of hospital stay, treatment intensity, 
and quality of life, as well as other patient/family centered outcomes. ${ }^{2}$ Quality improvement intervention for communication between healthcare providers and these patients with their families is an important focus for improving the quality of care and patient/family-centered outcomes. ${ }^{2}$

Several recent systematic reviews have focused on quality improvement in important aspects of palliative care including areas relevant to communication. In a 2008 review, Lorenz et al. identified studies supporting the effectiveness of a variety of communication interventions, particularly clarification of goals of care. ${ }^{3}$ In a 2008 review, Walling et al. found both similar results and also that broader end-of-life communication interventions improved patient satisfaction and psychological outcomes. ${ }^{4}$ Finally, a 2011 review of studies on communication in intensive care found evidence supporting the effectiveness of printed information and structured communication by the regular intensive care unit team, palliative care, and ethics consultations for the outcomes of distress and healthcare utilization. ${ }^{5}$ However, none of these studies focused on seriously ill patients across all settings, regardless of specialties, age, and disease conditions, or compared types of interventions.

A recent consensus report published by the Improving Palliative Care in the Intensive Care Unit group concluded that there are two categorizations for quality improvement in this setting: 'consultative models,' which involve consulting communication specialists, such as palliative medicine teams, in the care of patients and families, and 'integrative models,' which seek to embed palliative care principles and interventions into daily practice by the usual care team. ${ }^{6}$ These categories are not mutually exclusive as interventions may be both integrative and consultative. Regardless, for interventions targeting communication, we believe these categorizations are relevant across all care settings; integrative approaches involve structural changes in care and provider training, while consultative approaches incorporate additional trained palliative care or palliative care-related consultants to specifically address communication issues. ${ }^{7}$

Consequently, we conducted a systematic review to evaluate the effectiveness of quality improvement interventions focusing on communication for populations with advanced and serious illness across all settings including ambulatory care, acute medical care wards in hospitals, adult outpatient clinics, pediatric wards, pediatric outpatient clinics, oncology clinics/ wards, intensive care units, hospices, and nursing homes. We also compared whether communication quality improvement interventions that are integrative, as opposed to consultative, are more likely to impact patient and family outcomes.

\section{METHODS}

We searched PubMed, CINAHL, PsycINFO, Cochrane, and DARE for articles published from 2000 through December 2011 and identified additional studies from reference lists of eligible articles and relevant systematic reviews.

\section{Search Strategy}

Our electronic search strategy included medical subject headings (MeSH) and keywords related to palliative care, quality improvement, communication, cancer, terminally ill, hospice care, patient care planning, and quality assurance.

\section{Study Selection}

We included prospective, controlled studies that included a majority of patients that were seriously ill or had advanced disease and who were unlikely to be cured, recover, or stabilize. ${ }^{8}$ Seriously ill patients and those with advanced disease in pediatric, adult, and geriatric populations with all conditions (e.g., cancer, heart failure, end-stage lung disease, dementia, and frailty) were included. We included studies where the focus of the intervention was communication between providers and patients and/or families, and which measured the impact of the intervention on patient and/or family-centered outcomes, including satisfaction, quality of life, symptoms, and healthcare utilization. Since the nature of both quality improvement and palliative care practice has changed substantially since 2000 , and other previous reviews have addressed literature published prior to $2000,{ }^{3}$ we searched and included articles published between January 2000 and December 2011.

\section{Data Extraction and Quality Assessment}

Two reviewers independently screened first the abstract and then the full article for eligibility (Fig. 1). One reviewer abstracted data from included articles, with the work checked by a second reviewer. We abstracted population characteristics, study design, setting, and description of interventions, along with study outcomes and findings. We also abstracted population and intervention characteristics and the effect of interventions on the outcomes of interest: patient and family satisfaction, quality of life, healthcare utilization (as measured by decreased intensive care unit (ICU) or hospital length of stay, and/or other aggressive treatments), and symptom control. We also abstracted whether the interventions were integrative, consultative, or combined using the following definitions: integrative (embedding palliative care principles and interventions into daily practice, such as provider education or organizational strategies), and consultative (utilizing additional services, such as palliative care consultants).

To minimize errors, data abstraction was done by at least two independent reviewers. Data abstraction forms were also designed to target specific outcomes and were pilot tested prior to use. All evidence was graded for the strength of the best available evidence, including the risk of bias in relevant studies using the GRADE Working Group criteria adapted by the Agency for Healthcare Research and Quality (AHRQ); ${ }^{8,9}$ for details, see the report. ${ }^{10}$ 


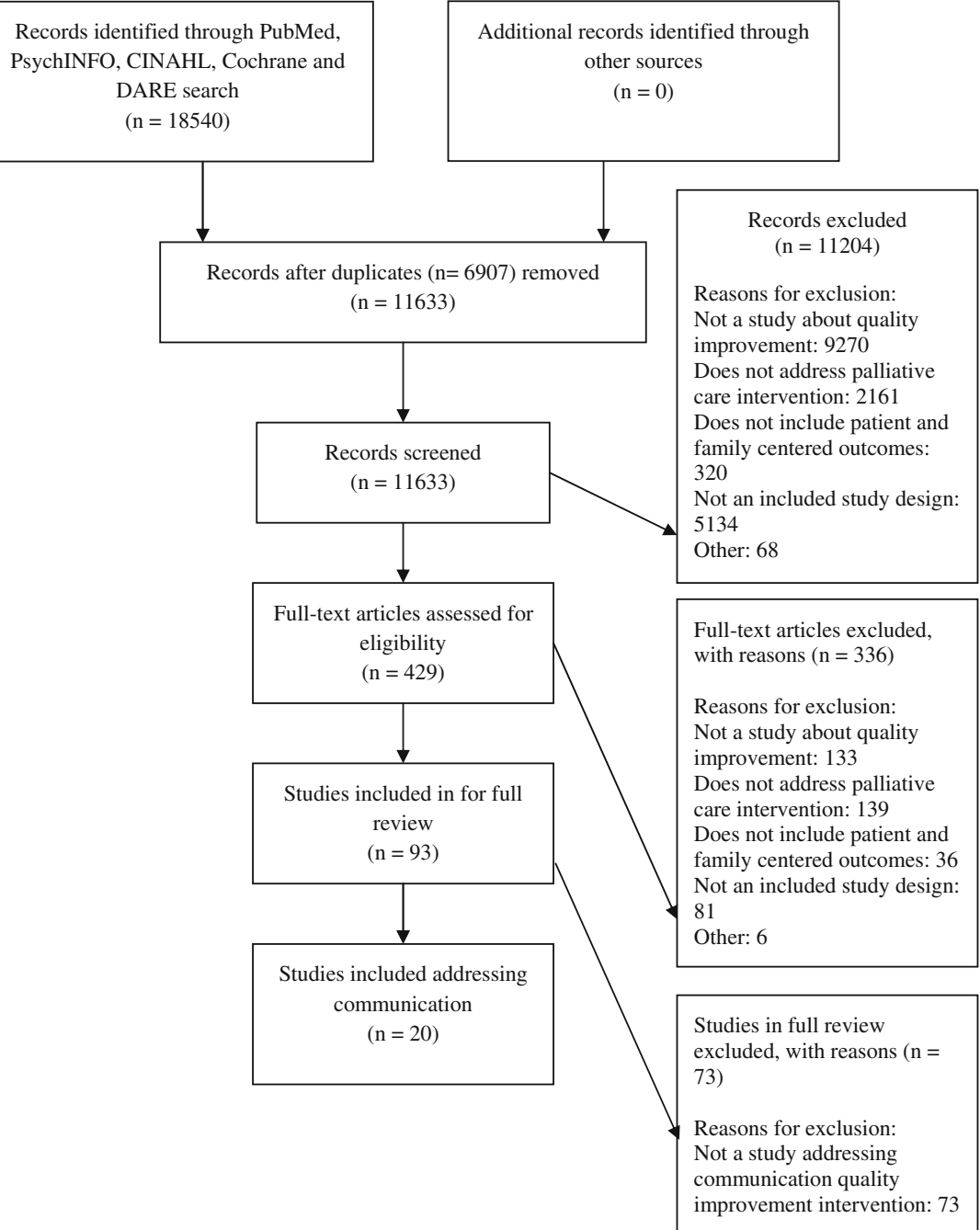

Figure 1. Flow diagram for study selection.

\section{Data Synthesis and Analysis}

We present qualitative summaries of individual studies. Due to intervention and outcome heterogeneity, meta-analyses could not be conducted.

\section{RESULTS}

\section{Study Characteristics}

Of the initial 11,633 titles and abstracts identified, 429 articles were screened, and 93 met the criteria for full review. Of these 93 articles, 20 met all of the inclusion criteria for quality improvement interventions targeting communication in patients with advanced and serious illness ${ }^{11-30}$ (Fig. 1). Sample sizes ranged from 63 to 2,891 patients with two-thirds of studies having between 150 and 900 patients (Table 1). In total, 13 of the 20 studies were conducted in an intensive care unit $^{11,12,14,18-21,23-26,28,29}$ with one of these in a neonatal ICU. ${ }^{20}$ The remaining seven studies were conducted in nonintensive care unit settings: ${ }^{13,15-17,22,27,30}$ one nursing home ${ }^{22}$ two ambulatory ${ }^{13,30}$ and four inpatient hospital units. ${ }^{15-17,27}$ Ten were multicenter studies, ${ }^{13-}$ $16,19,22,24,26,29,30$ and nine were randomized controlled trials. ${ }^{13,15,16,19,22,27-30}$ Of the 11 non-randomized controlled trials, ${ }^{11,12,14,17,18,20,21,23-26} 7$ were pre-post prospective studies conducted in the same setting with different sets of patients. ${ }^{12,14,20,21,23-25}$ All studies had a comparison group of usual care, except for one study where all patients were seen by palliative care physicians. ${ }^{13}$ Only one study ${ }^{26}$ reported on a very low birth weight pediatric population; the remaining 19 studies were in adult populations with mean age ranging from 40 to 87 years (Table 1). Most of the studies $(n=15)$ included patients with mixed illnesses. ${ }^{11,12,14-22,24,25,28,29}$ All of the studies used patient/family education as part of the intervention, and half used provider education. ${ }^{11,12,17,19,22,24,26-29}$ 
Table 1. Study Characteristics of Articles Addressing Communication

\begin{tabular}{|c|c|c|c|c|c|c|}
\hline Author, year & Study design & Study size & Setting & Center & Mean age & Disease populations \\
\hline Ahrens, $2003^{11}$ & Non-RCT & 151 & $\mathrm{ICU}$ & Single & Adult & Mixed illnesses \\
\hline Campbell, $2003^{12}$ & Non-RCT & 81 & ICU & Single & Adult & Mixed illnesses \\
\hline Clayton, $2007^{13}$ & $\mathrm{RCT}$ & 174 & Ambulatory & Multi-center & 65 & Mixed cancer \\
\hline Cowan, $2003^{14}$ & Non-RCT & 873 & $\mathrm{ICU}$ & Multi-center & 61 & Mixed illnesses \\
\hline Gade, $2008^{15}$ & RCT & 517 & Hospital & Multi-center & 73.6 & Mixed illnesses \\
\hline Hanks, $2002^{16}$ & $\mathrm{RCT}$ & 261 & Hospital & Multi-center & 68.4 & Mixed illnesses \\
\hline Jacobsen, $2011^{17}$ & Non-RCT & 899 & Hospital & Single & 62.9 & Mixed illnesses \\
\hline Kaufer, $2008^{18}$ & Non-RCT & 88 & $\mathrm{ICU}$ & Single & Adult & Mixed illnesses \\
\hline Laurette, $2007^{19}$ & RCT & 63 & $\mathrm{ICU}$ & Multi-center & 72 & Mixed illnesses \\
\hline Lilly, $2000^{20}$ & Non-RCT & 530 & $\mathrm{ICU}$ & Single & 59 & Mixed illnesses \\
\hline Lilly, $2003^{21}$ & Non-RCT & 2891 & ICU & Single & 59 & Mixed illnesses \\
\hline Molloy, $2000^{22}$ & RCT & 1133 & Nursing homes & Multi-center & 83.1 & Mixed illnesses \\
\hline Mosenthal, $2008^{23}$ & Non-RCT & 367 & ICU & Single & 40 & Trauma \\
\hline Muir, $2010^{24}$ & Non-RCT & 480 & $\mathrm{ICU}$ & Multi-center & 54 & Mixed illnesses \\
\hline Norton, $2007^{25}$ & Non-RCT & 191 & $\mathrm{ICU}$ & Single & 66.1 & Mixed illnesses \\
\hline Penticuff, $2005^{26}$ & Non-RCT & 154 & NICU & Multi-center & VLBW & Pediatric \\
\hline Sampson, $2011^{27}$ & $\mathrm{RCT}$ & 32 & Hospital & Single & 87 & Advanced dementia \\
\hline Schneiderman, $2000^{28}$ & $\mathrm{RCT}$ & 70 & $\mathrm{ICU}$ & Single & 49 & Mixed illnesses \\
\hline Schneiderman, $2003^{29}$ & $\mathrm{RCT}$ & 546 & $\mathrm{ICU}$ & Multi-center & 67.5 & Mixed illnesses \\
\hline Tulsky, $2011^{30}$ & RCT & 264 & Ambulatory & Multi-center & 61 & Mixed cancer \\
\hline
\end{tabular}

$V L B W$ very low birth weights, ICU intensive care unit, NICU neonatal intensive care unit

\section{Common Types of Interventions}

Despite the heterogeneity of the interventions, four common types emerged (Table 2).

1. Communication quality improvement intervention through family meetings: We included studies in this category that increased the frequency or structure of information sharing sessions with one or more of the patient's usual health providers, usually a physician, nurse, and/or social worker (not a palliative care team), with patients and families to improve communication on issues relevant to advanced and serious illness, such

Table 2. Outcomes of Studies by Type of Communication Improvement Interventions

\begin{tabular}{|c|c|c|c|c|c|c|c|}
\hline Author, year & Intervention type & $\begin{array}{l}\text { Integrative or } \\
\text { consultative }\end{array}$ & $\begin{array}{l}\text { Patient } \\
\text { satisfaction }\end{array}$ & $\begin{array}{l}\text { Family } \\
\text { satisfaction }\end{array}$ & $\begin{array}{l}\text { Healthcare } \\
\text { utilization }\end{array}$ & $\begin{array}{l}\text { Quality of } \\
\text { life }\end{array}$ & $\begin{array}{l}\text { Symptom } \\
\text { control }\end{array}$ \\
\hline Ahrens, $2003^{11}$ & Family meetings & Combined & & & Significant $^{\mathrm{a}}$ & & \\
\hline Cowan, $2003^{14}$ & Family meetings & Consultative & Not significant & & Significant $\mathrm{t}^{\mathrm{a}}$ & & \\
\hline Jacobsen, $2011^{17}$ & Family meetings & Integrative & & & Significant ${ }^{\mathrm{a}}$ & & \\
\hline Kaufer, $2008^{18}$ & Family meetings & Consultative & Not significant & Significant $^{\mathrm{a}}$ & & & $\begin{array}{l}\text { Not } \\
\text { significant }\end{array}$ \\
\hline Laurette, $2007^{19}$ & Family meetings & Combined & & & $\begin{array}{l}\text { Not } \\
\quad \text { significant }\end{array}$ & & Significant $^{\mathrm{a}}$ \\
\hline Lilly, $2000^{20}$ & Family meetings & Integrative & & & Significant $^{\mathrm{a}}$ & & \\
\hline Lilly, $2003^{21}$ & Family meetings & Integrative & & & Significant $\mathrm{t}^{\mathrm{a}}$ & & \\
\hline Molloy, $2000^{22}$ & Family meetings & Integrative & Not significant & Not significant & Significant $\mathrm{t}^{\mathrm{a}}$ & & \\
\hline Mosenthal, $2008^{23}$ & Family meetings & Combined & & & Significant $\mathrm{t}^{\mathrm{a}}$ & & \\
\hline Muir, 2010 & Family meetings & Integrative & & & Not significant & & \\
\hline Penticuff, $2005^{26}$ & Family meetings & Integrative & Not significant & & & & \\
\hline Campbell, $2003^{12}$ & $\begin{array}{l}\text { Palliative care } \\
\text { team }\end{array}$ & Consultative & & & Significant $^{\mathrm{a}}$ & & \\
\hline Gade, $2008^{15}$ & $\begin{array}{l}\text { Palliative care } \\
\text { team }\end{array}$ & Consultative & Significant $^{\mathrm{a}}$ & & Significant $^{\mathrm{a}}$ & $\begin{array}{l}\text { Not } \\
\quad \text { significant }\end{array}$ & $\begin{array}{l}\text { Not } \\
\text { significant }\end{array}$ \\
\hline Hanks, $2002^{16}$ & $\begin{array}{l}\text { Palliative care } \\
\text { team }\end{array}$ & Consultative & Not significant & & Not significant & $\begin{array}{l}\text { Not } \\
\text { significant }\end{array}$ & $\begin{array}{l}\text { Not } \\
\text { significant }\end{array}$ \\
\hline Norton, $2007^{25}$ & $\begin{array}{l}\text { Palliative care } \\
\text { team }\end{array}$ & Consultative & & & Not significant & & \\
\hline Sampson, $2011^{27}$ & $\begin{array}{l}\text { Palliative care } \\
\text { team }\end{array}$ & Combined & & Not significant & & & \\
\hline $\begin{array}{l}\text { Schneiderman, } \\
2000^{28}\end{array}$ & Ethics consultation & Consultative & & & Significant $^{\mathrm{a}}$ & & \\
\hline $\begin{array}{l}\text { Schneiderman, } \\
2003^{29}\end{array}$ & Ethics consultation & Consultative & & & Significant $^{\mathrm{a}}$ & & \\
\hline Clayton, $2007^{13}$ & $\begin{array}{c}\text { Physician-patient } \\
\text { communication }\end{array}$ & Integrative & Not significant & & & & \\
\hline Tulsky, $2011^{30}$ & $\begin{array}{l}\text { Physician-patient } \\
\text { communication }\end{array}$ & Integrative & Not significant & & & & \\
\hline
\end{tabular}

${ }^{a}$ Significant in favor of the intervention group 
as goals of care. Eleven studies examined the efficacy of various types of family meetings to improve communication in palliative care. ${ }^{11,14,17-24,26}$ Two of these 11 studies were RCTs. ${ }^{19,22}$ Interventions grouped in this category focused either on increasing the frequency of these meetings, increasing by using an active communication strategy including elements such as detailed communication guidelines, longer conferences and more time for family members to discuss with healthcare staff, or training and education of providers to improve provider-patient/family communication. Nine studies using the family meeting approach as the intervention to improve communication evaluated healthcare utilization as an outcome, and seven of these nine studies $(77 \%)$ found statistically significant improvements in healthcare utilization. ${ }^{11,14,17,20-23}$,

2. Communication quality improvement intervention through interdisciplinary palliative care teams: Studies in this category used a palliative care team intervention where the primary focus was communication to improve patients' and families' understanding of the medical condition and treatment decisions. The palliative care team may include a palliative care physician and nurse, hospital social worker, and/or chaplain, interacting with the patient/family and usual care clinical team to address issues such as patient symptoms, diagnosis, prognosis, and goals of care. Five studies $^{12,15,16,25,27}$ used this style of intervention to improve communication to facilitate decision making on issues such as goals of care and potential treatment limitations. Four studies out of these five evaluated healthcare utilization as an outcome, and two (50\%) of the four studies found a statistically significant effect on healthcare utilization (fewer ICU admissions and reduced use of non-beneficial aggressive treatment). ${ }^{12,15}$

3. Ethics consultations: Ethics consultation is defined as the use of specialized help in identifying, analyzing, and resolving ethical problems that arise in the care of the patient. ${ }^{31}$ Studies in this category used ethics consultation as a communication quality improvement intervention to communicate with providers, patients, and families about ethical issues and address conflicts about life-sustaining treatments. Two studies ${ }^{28,29}$ examined the impact of ethics consultations by comparing outcomes for patients randomly assigned to an intervention offering ethics consultation to the control group not offered ethics consultation. Both (100\%) of these studies (a single-center RCT followed by a multicenter RCT of the same intervention) demonstrated significant decreases in both ICU length of stay and the number of days that aggressive interventions, such as artificial nutrition, hydration, and ventilator treatments, were used.

4. Physician-patient communication quality improvement intervention: Two studies were in this category. ${ }^{13,30}$
The first study $^{13}$ evaluated the use of a question prompt list for patients with advanced cancer receiving palliative care consultation to assist patients to formally ask questions about end-of-life issues and to improve patient-physician communication around prognosis. Although the intervention significantly increased communication on these issues, there was no statistically significant impact on patient satisfaction. For the other study ${ }^{30}$, physicians in the intervention group viewed a 1-h lecture on communication skills delivered by one of the investigators and used a CD-ROM training program on communication skills that was tailored with examples from clinic visits. The interactive CD-ROM training program on communication skills was designed to enhance physician's ability to respond effectively to patients' emotional concerns. The intervention was based on social cognitive theory and had five modules: principles of effective communication, recognizing empathic opportunities, responding to empathic opportunities, conveying prognosis, and answering difficult questions. A final module summarized main points from the intervention. Physicians in the intervention group also received financial incentives and a pair of high-quality headphones, while the control group received no training beyond the 1-h lecture. In this study, the computerized intervention improved the quality of communication in medical encounters and the control physicians performed slightly worse than the intervention group in the post-intervention phase. However, there was no statistically significant effect on patient satisfaction.

\section{Consultative vs. Integrative Interventions}

Interventions from four studies were combined, including both integrative and consultative components. ${ }^{11,19,23,27}$ Eight interventions were integrative; $;^{13,17,20-22,24,26,30}$ five of these were non-RCTs, ${ }^{17,20,21,24,26}$ and three were highquality RCTs. ${ }^{13,22,30}$ Four of the eight $(50 \%)$ had a significant improvement in key outcomes in the intervention group as compared to the control group. ${ }^{17,20-22}$ Eight interventions were consultative in nature, ${ }^{12,14-16,18,25,28,29}$ six of the eight $(75 \%)$ had a significant improvement in at least one key outcome due to the intervention. ${ }^{12,14,15,18,28,29}$ Four of these were non-RCTs, and four were mediumquality RCTs (Table 1).

\section{Outcomes Results}

Results are categorized below by outcome; some studies evaluated the impact of the intervention on more than one outcome (Table 2). 


\section{Patient and Family Satisfaction}

Nine studies evaluated patient and/or family satisfaction; two of these nine studies (22\%) found statistically significant improvement on patient and/or family satisfaction. ${ }^{13-16,18,22,26,27,30}$ Eight of the nine studies evaluated patient satisfaction as an outcome. ${ }^{13-}$ 16,18,22,26,30 Five of these eight studies were RCTs of high quality, ${ }^{13,15,16,22,30}$ with the remaining three studies being non-RCTs of medium quality. ${ }^{14,18,26}$ Only one RCT found a statistically significant improvement on patient satisfaction with the intervention; ${ }^{15}$ it used an interdisciplinary palliative care service to address patient and family needs for information and decision making on goals of care.

Three of the nine studies assessed the intervention effect on family satisfaction. Two RCTs, one with a large sample $\operatorname{size}^{22}(n=1,133)$ and a smaller RCT with a small sample $\operatorname{size}^{27}(n=32)$, both showed no significant improvement in family satisfaction. The third study, a non-RCT with a small sample size $(n=88)$ showed significant improvement with the intervention in family satisfaction. ${ }^{18}$

\section{Healthcare Utilization}

Fifteen studies evaluated healthcare utilization as an outcome, and 11 studies $(73 \%)$ found statistically significant improvement on this outcome. Six of the 15 studies were RCTs of medium quality, ${ }^{15,16,19,22,28,29}$ and 4 of these studies $(67 \%)$ showed a statistically significant improvement attributed to the intervention. ${ }^{15,22,28,29}$ Nine of the 15 studies were non-RCTs, ${ }^{11,12,14,17,20,21,23-25}$ and 7 of these studies (78\%) showed a statistically significant improvement in healthcare utilization. ${ }^{11,12,14,17,20,21,23}$ Sample size, intervention type, or setting did not explain the difference between the studies with significant and non-significant results.

\section{Quality of Life (QOL) and Symptom Control}

Two high-quality RCTs assessed the QOL of patients. ${ }^{15,16}$ They both showed no significant improvement on QOL in the intervention as compared to the usual care group (Table 2). These two studies independently used the same type of intervention among different sample populations in similar settings (Table 2). Four studies evaluated symptom control of patients: three of these four were high-quality RCTs, ${ }^{15,16,19}$ and the last was a medium-quality non-RCT. ${ }^{18}$ Only one of these four studies ( $25 \%$ ), a RCT, ${ }^{19}$ showed a statistically significant improvement in symptom control; it also had the smallest sample size $(n=63)$ of all the studies included in this review (Table 1 and Table 2).

\section{DISCUSSION}

In conclusion, we identified 20 studies targeting communication in patients with advanced and serious diseases, including a number of high-quality RCTs. Most interventions addressed adult mixed-illness populations, predominantly in an intensive care unit setting, and comprised either improvements in family meetings or better utilization of palliative care consultations. Nearly three-quarters of the interventions that evaluated healthcare utilization outcomes, such as intensive care unit length of stay and potentially non-beneficial life-sustaining aggressive treatment, found statistically significant effects of the intervention. In studies that evaluated intervention impact on the outcomes of satisfaction, quality of life, and symptom control, findings were predominantly not significant. Although the number of included studies is small, a higher proportion of consultative studies found a statistically significant improvement because of the intervention. This could suggest a trend that consultative interventions, as opposed to integrative ones, might be more effective; however, more research is needed on this topic. Healthcare utilization was the most commonly reported outcome in these studies, and threequarters of studies measuring this outcome showed a significant effect because of the intervention. Optimizing healthcare utilization is important, especially in intensive care units where beds are often a limited resource and rising healthcare costs associated with end-of-life care are an increasing concern. However, although better healthcare utilization may reflect improved decision-making and reduced patient suffering and family burden, the current evidence does not support a strong correlation between that utilization and other patient/family-centered outcomes. ${ }^{32}$ In our analysis, few studies measured patient/ family satisfaction, quality of life, or symptoms, and almost none found statistically significant improvements in these outcomes. In part, this may be because the outcomes of improved communication are difficult to measure; the outcome of "satisfaction" likely reflects patient and family experiences beyond those impacted by communication. Development of better instruments, which more precisely capture and reflect the impact of good communication, will facilitate the accurate evaluation of communication-related interventions.

Most of these studies also focused on increasing levels of communication or structuring of communication rather than improving use of specific communication models. Patientcentered communication is a complex task, particularly around end-of-life decision making, and requires a variety of elements and provider skills, including cultural awareness, building partnership, eliciting key information, providing evidence, and checking for understanding; ${ }^{33}$ structuring any intervention to improve this multi-faceted task is understandably difficult. 
No previous studies, to our knowledge, have addressed whether the type of intervention-consultative vs. integrative - may impact intervention efficacy. Our study supports that consultative interventions, as opposed to integrative ones, are more likely to potentially improve healthcare utilization, although there are successful examples of both interventions types. This benefit could be due to multiple potential reasons. Consultative teams may focus specifically on communication-related issues as opposed to balancing them with other clinical responsibilities as must occur with integrative interventions. Also, these interventions inherently involve culture change, and, as outside agents of such change, consultative interventions may be more reliably and/or rapidly effective. Finally, the studies, with a focus on communication, showed little evidence for effect on overall symptoms or quality of life: this may relate to the focus of these interventions, although other reviews of broader palliative care interventions have also not found impact on these outcomes. ${ }^{3}$

Our review has some limitations. Although all studies focused on improving communication, the heterogeneity in populations, interventions, outcomes, and measurement tools did not allow for a quantitative synthesis of the literature. Because we limited our review to studies with populations where a majority of patients met the definition of seriously ill and/or with advanced disease, we excluded some studies with potentially relevant interventions in broader populations; however, many of these studies have been included in other more general systematic reviews. ${ }^{4,5}$ We also included only studies that focused on communication and consequently multi-faceted studies that included communication as one of multiple targets were not addressed; see report for full details on these studies. ${ }^{10}$ Finally, we only abstracted outcomes that were patient/ family-centered and reported in at least two of the studies; consequently, several outcomes (e.g., family symptoms and staff assessments of quality of care) are not summarized in this review.

The literature in this area also has a number of limitations. As studies often reported many outcomes and rarely designated primary outcomes, interpretation of results was difficult, and study outcomes were diluted (e.g., reporting on a long list of outcomes rather than focusing on those most likely to be impacted by the intervention). The quality of many studies was medium to low based on the risk of bias tools (GRADE Working Group criteria adapted by $\mathrm{AHRQ}^{8,9}$ ) in which a low risk of bias was assessed if six or more of the items were scored as a "yes," a medium risk of bias was assessed if four or five of the items were scored as a "yes," and a high risk of bias was assessed if zero to three items were scored as a "yes." Common quality issues included: small sample sizes, often due to insufficient recruitment and high rates of attrition, and outcome measures not designed for advanced disease or end-of-life populations and/or not well coordinated with the purpose of the intervention. Limited descriptions of interventions often lacked detailed information of both what the intervention entailed and in which context it was implemented, and effectiveness may have been influenced by unmeasured factors, such as individual team member characteristics and experience and contextual issues in individual institutions. Furthermore, RCTs often did not report key elements, such as blinding or allocation, which lowered scores in the grading of the evidence. None of the studies compared or tested different intervention methods, timing, or participants. There were no studies in hospice populations, only one pediatric study (in a neonatal ICU), and a few in the outpatient setting. None of the studies focused on either cultural issues in communication at the end of life or on reducing disparities in outcomes; these are areas where there are significant needs for, but challenges in, developing and evaluating interventions.

In summary, in this systematic review of studies focusing on communication in end-of-life populations, we found moderate evidence for the impact of quality improvement interventions on reducing healthcare utilization, particularly in the intensive care unit setting. Consultative interventions may be more likely to improve healthcare utilization. Multiple studies evaluated patient and/or family satisfaction, but only two showed statistically significant improvements attributed to the intervention. Elements that should be considered for future studies include: better development and description of interventions, inclusion of theory and quality improvement techniques, consideration of context, and matching outcomes to interventions. Development of measurement instruments is needed for measuring certain domains and for better understanding how to match outcome measures to interventions in order to effectively measure the impact of these interventions on outcomes other than healthcare utilization. Further research is needed on: impacting communication outcomes other than healthcare utilization; establishing links between healthcare utilization and patient/family-centered outcomes; exploring other aspects of communication, such as cultural issues; improving communication in pediatric populations; and improving communication outside of the intensive care setting.

Acknowledgments: This project was funded under contract no. HHSA-290-2007-10061-I-EPC3 from the Agency for Healthcare Research and Buality, US Department of Health and Human Services. The Agency for Healthcare Research and Quality reviewed contract deliverables for adherence to contract requirements and quality and provided a copyright release for submission. The authors of this report are responsible for its content. Statements in the report should not be construed as endorsement by the Agency for Healthcare Research and Quality or the US Department of Health and Human Services. 
The authors want to thank Dr. Supriya Janakiraman and Dr. Judy Sangl for their assistance as the task order officer on the original contract and reviewing the manuscript. Fawole OA, Dy SM, Wilson R, Lau BD, Martinez K, Apostol C, Vollenweider D, Bass EB, and Aslakson $R$ contributed to the study concept, design, analysis, interpretation, and preparation of the manuscript.

Conflict of Interest: The authors declare that they do not have a conflict of interest.

Corresponding Author: Oluwakemi A. Fawole, MBChB, MPH; Johns Hopkins University Evidence-Based Practice Center, 1830 East Monument Street, Room 8065, Baltimore, MD 21287, USA (e-mail: ofawole1@jhmi.edu).

\section{REFERENCES}

1. Gattellari M, Voigt KJ, Butow PN, Tattersall MH. When the treatment goal is not cure: are cancer patients equipped to make informed decisions? J Clin Oncol. 2002;20(2):503-513.

2. Curtis JR, Patrick DL, Shannon SE, Treece PD, Engelberg RA, Rubenfeld GD. The family conference as a focus to improve communication about end-of-life care in the intensive care unit: opportunities for improvement. Crit Care Med. 2001;29(2 Suppl):N26-N33.

3. Lorenz KA, Lynn J, Dy SM, et al. Evidence for improving palliative care at the end of life: a systematic review. Ann Intern Med. 2008;148(2):147-159.

4. Walling A, Lorenz KA, Dy SM, et al. Evidence-based recommendations for information and care planning in cancer care. J Clin Oncol. 2008;26 (23):3896-3902.

5. Scheunemann LP, McDevitt M, Carson SS, Hanson LC. Randomized, controlled trials of interventions to improve communication in intensive care: a systematic review. Chest. 2011;139(3):543-554.

6. Nelson JE, Bassett R, Boss RD, et al. Models for structuring a clinical initiative to enhance palliative care in the intensive care unit: a report from the IPAL-ICU Project (Improving Palliative Care in the ICU). Crit Care Med. 2010;38(9):1765-1772.

7. National Consensus Project for Quality Palliative Care (NCP). Clinical Practice Guidelines for Quality Palliative Care, Second Edition; 2009.

8. Methods Guide for Effectiveness and Comparative Effectiveness Reviews: AHRQ Publication No. 10(11)-EHC063-EF. Rockville, MD: Agency for Healthcare Research and Quality. March 2011. [Cited 2012 Jul 25]. Available at: http://effectivehealthcare.ahrq.gov/ehc/products/60/ 318/MethodsGuide_Prepublication-Draft_20120523.pdf.

9. Atkins D, Chang S, Gartlehner G et al. Assessing the Applicability of Studies When Comparing Medical Interventions. Agency for Healthcare Research and Quality; December 2010. Chapter 6 of Methods Guide for Comparative Effectiveness Reviews. AHRQ Publication No. 11 EHC019-EF. [Cited 2012 July 25]. Available at: http://www.effective healthcare.ahrq.gov/tasks/sites/ehc/assets/File/Methods_Guide Atkins.pdf.

10. Dy SM, Aslakson RA, Wilson RF et al. Interventions to Improve Health Care and Palliative Care for Advanced and Serious Illness. Closing the Quality Gap: Revisiting the State of the Science. Evidence Report No. 208. (Prepared by the Johns Hopkins University Evidence-based Practice Center under Contract. No. Contract No. 290-2007-10061-I.) AHRQ Publication No. 12-E014-EF. Rockville, MD: Agency for Healthcare Research and Quality. October 2012. www.effectivehealthcare.ahrq.gov/reports/final.cfm.

11. Ahrens T, Yancey V, Kollef M. Improving family communications at the end of life: implications for length of stay in the intensive care unit and resource use. Am J Crit Care. 2003;12(4):317-323. discussion 324.

12. Campbell ML, Guzman JA. Impact of a proactive approach to improve end-of-life care in a medical ICU. Chest. 2003;123(1):266-271.
13. Clayton JM, Butow PN, Tattersall MH, et al. Randomized controlled trial of a prompt list to help advanced cancer patients and their caregivers to ask questions about prognosis and end-of-life care. J Clin Oncol. 2007;25(6):715-723.

14. Cowan JD, Burns D, Palmer TW, Scott J, Feeback E. A palliative medicine program in a community setting: 12 points from the first 12 months. Am J Hosp Palliat Care. 2003;20(6).

15. Gade G, Venohr I, Conner D, et al. Impact of an inpatient palliative care team: a randomized control trial. J Palliat Med. 2008;11(2):180190.

16. Hanks GW, Robbins M, Sharp D, et al. The IMPACT study: a randomized controlled trial to evaluate a hospital palliative care team. $\mathrm{Br} \mathrm{J}$ Cancer. 2002;87(7):733-739.

17. Juliet J, Ellen R, Jackson VA, Meigs JB, Andrew BJ. Development of a cognitive model for advance care planning discussions: results from a quality improvement initiative. J Palliat Med. 2011;14(3):331-336.

18. Kaufer M, Murphy $\mathbf{P}$, Barker K, Mosenthal A. Family satisfaction following the death of a loved one in an inner city MICU. Am J Hosp Palliat Care. 2008;25(4):318-325.

19. Lautrette A, Darmon M, Megarbane B, et al. A communication strategy and brochure for relatives of patients dying in the ICU. N Engl J Med. 2007;356(5):469-478.

20. Lilly CM, De Meo DL, Sonna LA, et al. An intensive communication intervention for the critically ill. Am J Med. 2000;109(6):469-475.

21. Lilly CM, Sonna LA, Haley KJ, Massaro AF. Intensive communication: four-year follow-up from a clinical practice study. Crit Care Med. 2003;31(5 Supplement):S394-S399.

22. Molloy DW, Guyatt GH, Russo R, et al. Systematic implementation of an advance directive program in nursing homes: a randomized controlled trial. JAMA. 2000;283(11):1437-1444.

23. Mosenthal AC, Murphy PA, Barker LK, Lavery R, Retano A, Livingston DH. Changing the culture around end-of-life care in the trauma intensive care unit. J Trauma. 2008;64(6):1587-1593.

24. Muir JC, Daly F, Davis MS, et al. Integrating palliative care into outpatient private practice oncology setting. J Pain Symptom Manag. 2010;40(1): 126-135.

25. Norton SA, Hogan LA, Holloway RG, Temkin-Greener H, Buckley MJ, Quill TE. Proactive palliative care in the medical intensive care unit: effects on length of stay for selected high-risk patients. Crit Care Med. 2007;35(6):1530-1535.

26. Penticuff $\mathbf{J H}$, Arheart KL. Effectiveness of an intervention to improve parent-professional collaboration in neonatal intensive care. $\mathrm{J}$ Perinat Neonatal Nurs. 2005;19(2):187-202.

27. Sampson EL, Jones L, Thuné-Boyle IC, et al. Palliative assessment and advance care planning in severe dementia: an exploratory randomized controlled trial of a complex intervention. Palliat Med. 2011;25 (3):197-209. Epub 2011 Jan 12.

28. Schneiderman LJ, Gilmer T, Teetzel HD. Impact of ethics consultations in the intensive care setting: a randomized, controlled trial. Crit Care Med. 2000;28(12):3920-3924.

29. Schneiderman LJ, Gilmer T, Teetzel HD, et al. Effect of ethics consultations on non-beneficial life-sustaining treatments in the intensive care setting: a randomized controlled trial. JAMA. 2003;290 (9): 1166-1172.

30. Tulsky JA, Arnold RM, Alexander SC, et al. Enhancing communication between oncologists and patients with a computer-based training program: a randomized trial. Ann Intern Med. 2011;155 (9):593-601.

31. Fletcher JC, Quist N, Jonsen AR. Ethics consultation in health care. Health Administration Press; 1989 ISBN 0910701393.

32. Fenton JJ, Jerant AF, Bertakis KD, Franks P. The cost of satisfaction. A national study of patient satisfaction, health care utilization, expenditures, and mortality. Arch Intern Med. 2012;172(5):405-411.

33. Epstein RM, Alper BS, Quill TE. Communicating evidence for participatory decision making. JAMA. 2004;291(19):2359-2366. 